\title{
THE FUNCTION OF CONSCIOUSNESS IS TO GENERATE EXPERIENCE
}

\author{
Axel Cleeremans ${ }^{1}$ \\ Catherine Tallon-Baudry ${ }^{2}$ \\ 1. Consciousness, Cognition \& Computation Group, Center for Research in Cognition \& Neuroscience, ULB \\ Neuroscience Institute, Université libre de Bruxelles (Belgium) \\ 2. Cognitive and computational neuroscience Lab, , Inserm, , École Normale Supérieure - PSL University, Paris \\ (France)
}

\begin{abstract}
Why would we do anything at all if the doing was not doing something to us? In other words: What is consciousness good for? Here, reversing classical views, according to many of which subjective experience is a mere epiphenomenon that affords no functional advantage, we propose that the core function of consciousness is precisely to enable subject-level experience. "What it feels like" is endowed with intrinsic value, and it is precisely the value agents associate with their experiences that explains why we do certain things and avoid others. Thus, we argue that it is only in virtue of the fact that conscious agents experience things and care about those experiences that they are motivated to act in certain ways and that they prefer some states of affairs vs. others. In this sense, conscious experience functions as a mental currency of sorts, which not only endows mental states with intrinsic value, but also makes it possible for conscious agents to compare vastly different experiences in a common subject-centered space - a feature that readily explains the fact that consciousness is unified. If, as we argue, the function of consciousness is to endow agents with subjective experience, then the hard problem of consciousness seems to dissolve.
\end{abstract}




\section{THE FUNCTION OF CONSCIOUSNESS IS TO GENERATE EXPERIENCE}

\section{What is the function of consciousness?}

What is the function of consciousness, if any? This fundamental question has thus far been addressed mostly by philosophers (e.g., Block, 1995; Kriegel, 2004; Tye, 1996). Surprisingly few things have been written about the functions of consciousness in the neuroscientific or psychological literature, perhaps because it is such a thorny issue. Seth (2018) provides a good overview of the manner in which the possible functions of consciousness have been approached. First, Seth points out, one need to consider the possibility that consciousness simply has no function whatsoever. This perspective is logically possible and amounts either to defend conscious inessentialism - the view that any intelligent activity that is carried out with consciousness can also be carried out without consciousness, and epiphenomenalism - the view that consciousness, while it exists, does not and cannot play any causal role beyond that played by its biological foundations. Different strands of research support such views.

For instance, the stupefying advances of artificial intelligence research are suggestive that feeling things is simply unnecessary to carry out complex information processing. Indeed, AI research continues to demonstrate that, in machines at least, superhuman performance is possible in the (obvious) absence of consciousness. In this respect, the recent demonstration that deep learning methods can master complex games such as a go or chess (Silver et al., 2018; Silver et al., 2017) without human input is particularly remarkable. But even a simple calculator can perform complex arithmetic operations unconsciously - something that no human can achieve without awareness. The feats of AI, as impressive as they are, are also a reminder that intelligence is not consciousness.

Further, the extensive body of research dedicated to documenting the extent of unconscious cognition (e.g., Hassin, Uleman, \& Bargh, 2005) has likewise sought to demonstrate that one can carry out all sorts of complex information processing in the absence of phenomenal experience, thereby questioning the very function of consciousness. Perhaps the paradigmatic example of such empirical research is blindsight (Weiskrantz, 1986), a pathological condition resulting from extensive damage to $\mathrm{V} 1$ in which participants report being blind yet remain able to make appropriate visual decisions. Blindsight indeed suggests that phenomenal experience is not always necessary to drive successful action, but one should not forget here that blindsight is a very unusual clinical condition. In most healthy participants, consciousness cannot simply be turned off - a critical yet obvious point that is often side-lined by researchers interesting in unconscious cognition.

However, it is worth noting a substantial change in perspective over the last decade about the extent to which mental operations are actually possible in the absence of awareness. There is a long history about research on unconscious cognition that we will not develop further here, but it is fair to say that the pendulum is now slowly swinging back to a state where one can again doubt that complex information processing can take place unconsciously (e.g., see Newell \& Shanks, 2014; Peters, Kentridge, Phillips, \& Block, 2017; Peters \& Lau, 2015; Phillips, 2021; Shanks, Green, \& Kolodny, 1994). This renewed skepticism stems in part from failures to replicate previous work, particularly in social psychology (e.g., Doyen, Klein, Pichon, \& Cleeremans, 2012; O'Donnell et al., 2018), but also from changing perspectives on the links between conscious and unconscious processing (Cleeremans, 2014; Searle, 1992). 
While the extent and limits of unconscious cognition thus remains very much debated, it seems to us simply undeniable that consciousness enables us to do things that we could not do otherwise. What are these things - the functions of consciousness - then? Seth delineates various putative such functions, essentially volition, rational action, flexibility, and integration. Likewise, Baars (1988) lists nine putative functions of consciousness (e.g., adaption and learning, decision-making or executive function, metacognitive and self-monitoring function, etc.). Strikingly, however, there is little discussion of subjective experience per se in either Baars' or Seth's analyses: What difference does it make to feel things? What functions could subjective experience itself have?

Perhaps this strange omission stems from the fact that modern characterizations of consciousness have often tended to strictly distinguish the putative functions of consciousness from its phenomenal aspects. This distinction is in fact precisely the distinction between accessconsciousness and phenomenal consciousness introduced by Block (1995): Accessconsciousness (A-consciousness) refers to the various functions that consciousness enables, such as global availability, verbal report, reasoning, and executive control. Phenomenal consciousness (P-consciousness), on the other hand, refers to the fact that we experience conscious mental states: there is something it is like for us to be in a specific conscious mental state; there is something it is like for a bat to be chasing insects at dusk; there is nothing it is like for Alpha Go (Silver et al., 2017) to win against Lee Sedol. The distinction is also at the core of Chalmers' hard problem (Chalmers, 1995). One can imagine, argue both Chalmers and Block, that the functions of consciousness can be wholly dissociated from its phenomenal aspects. On the one hand, there seems to be functionless phenomenal experiences - Block (1995) mentions orgasm as an example of a state that is associated with felt qualities yet seems (intrinsically) to be void of functions. On the other hand, one can imagine zombies who function exactly like we do, but without experiencing anything. These different arguments and empirical demonstrations, however, introduce a quandary. If phenomenal experience is but a mere epiphenomenon, a benign and causally inefficacious "user illusion" (Dennett, 2016, 2019; Frankish, 2016) why is it there at all? Is it nothing but a mere accident of evolution (Robinson, Maley, \& Piccinini, 2015)? And if phenomenal experience has a function after all, what is it? Addressing this quandary is the main goal of this article.

We begin by arguing that the distinction between access- and phenomenal consciousness, as important as it has been in shaping consciousness research, is in fact misleading, precisely because it dissociates function from phenomenal experience. Instead, we claim that the core function of consciousness is precisely to endow agents with experiences. In other words, what consciousness does is to make it possible for an agent to experience things. On the face of it, it seems rather absurd to assume that something that appears as important to us as our ability to experience things would serve no purpose at all and be a mere epiphenomenon. Most of what we do is in fact driven precisely by the experiences we seek to experience and by those we seek to avoid. In this light, Siewert (1998) asked whether one would accept zombification in exchange for a reward. Imagine, asks Siewert, that you would be able to carry out life exactly as you do now, except that you wouldn't feel anything at all. There would not be anything it is like to be you anymore, yet you would remain functionally identical to what you are now. Would you choose to be zombified if offered a big enough reward to do it? Most likely, you would refuse any such offer, except perhaps if by doing so you would save the life of a loved one or help address any other similar subjectively essential life-and-death situation. 
And indeed, what would be the point of doing anything at all if the doing wasn't doing something to us? What makes life worth living, it seems obvious to us, is precisely the fact that we experience things. And from this, it follows that phenomenal experience has intrinsic value - it is worth something for us - and probably worth quite a lot since, following Siewert's thought experiment, no monetary reward would be large enough for us to accept to be zombified. But, crucially, things that have value also typically play functional roles in our behaviour. Thus, phenomenal experience cannot be a mere epiphenomenon: it is endowed with intrinsic value, and that is what makes it functionally relevant.

Before we attempt to unpack this argument, let us make three important points that will serve as background assumptions for the rest of our analysis.

First, it is essential here to distinguish between conscious experience and mere sensitivity - a slice of cortex, a thermostat, a computer, even a plant — all exhibit sensitivity to particular states of affairs, but few would be willing to ascribe any form of consciousness to them. Awareness is not sensitivity. Thus, we assume that awareness minimally entails that an agent is sensitive to its own sensitivity; in other words, that one knows that one knows. This perspective espouses higher-order (HOT) views of consciousness (Brown, Lau, \& LeDoux, 2019; Lau \& Rosenthal, 2011; Rosenthal, 1997), but is also broadly consistent with Global Workspace Theory (GWT) (Dehaene, Kerszberg, \& Changeux, 1998) in the sense that both frameworks assume that mere first-order representations are never sufficient to yield conscious experience. If subjective experience - what it feels like - has a function, it must therefore be a function or a set of functions that go beyond mere sensitivity.

Second, our claim that phenomenal experience has intrinsic value only makes sense if agents are able to learn about which phenomenal states they want to find themselves in (Cleeremans, 2011; Cleeremans et al., 2020). As Dennett put it (personal communication), "How do we go from doing things for reasons to having reasons for doing things?". Having reasons for doing things is precisely what differentiates conscious agents from agents such as Alpha Go (Silver et al., 2017), which, despite exhibiting superhuman skill when doing things, remains unable to do so for reasons of its own.

Third, this crucially links conscious experience with agenthood (Bayne, 2013; Pacherie, 2008). There is no sense in which we can talk about conscious experiences without first assuming there is an experiencer who experiences those experiences. The very notion of conscious experience presupposes the existence of a subject it is the experience of. As Frege (Frege, 1918) pointed out, "It seems absurd to us that a pain, a mood, a wish, should rove about the world without a bearer, independently. An experience is impossible without an experiencer. The inner world presupposes the person whose inner world it is." (p. 299). Merely sensitive systems do not care about the states they find themselves in for they lack a sense of their own existence as autonomous agents.

In the following, we explore these ideas, offering mostly discussion and (informed) speculation. We begin by discussing the surprisingly complex construct of value.

\section{The intrinsic value of subjective experience}

Consider the difference between being fed through an intravenous perfusion vs. eating a meal - even an unpalatable hospital meal. While both feeding methods serve the same physiological function, that is, to restore appropriate blood nutrients levels, consciously experiencing the 
taste, smell, and texture of food, as well as engaging in the act of eating, seems to be endowed with a distinctive value (Berthoud, 2011). This contrast suggests that subjective experience has intrinsic value, that is, that we value it for itself. As Lee (2019) puts it "Something has intrinsic value just in case that thing is good for its own sake". Zimmerman (2019) makes the point more precise: "whatever is intrinsically good is non derivatively good", by which he means that some things, such as money, only have value because they make it possible to obtain other things that you desire. Thus, the value of money is not intrinsic because its value is only derivative with respect to something else (i.e., it has instrumental value). And indeed, what would be the point of having money at all if all you could do with it was to see numbers go up on your bank account? The same analysis applies in vastly different domains, such as altruistic behaviour. For instance, as Zimmerman discusses, you may consider that helping people who need help is good. If prompted to explain why you think doing so is good, you might say that it is good to see people's needs be satisfied. If again asked why this is good, you might end up saying, as Zimmerman writes, "It just is good that people be pleased", at which point we seem to be unable to continue this derivation and conclude that satisfying one's needs is intrinsically good. We propose that subjective experience has, in this sense, intrinsic value. We will elaborate somewhat on the dimension(s) of value in the following, but it is worth underlining that in our view, value can be positive or negative.

Different philosophers have developed different views about the value of consciousness. In his analysis of the question, Lee (2019) defends what he calls the "neutral view", which he opposes to a "positive view" instantiated by both Siewert' (1998) and Seager's (2001) arguments that consciousness is intrinsically valuable. First, says Lee, the positive view is too liberal, for it merely defines intrinsic value in terms of instances thereof: Consciousness is valuable because there are experiences worth having. But if this were the case, then existence itself would be valuable, which is a non-trivial claim (that we will not discuss further here). In addition, says Lee, there are also instances of the disvalue of consciousness - imagine a situation where the only experience you can have is that of constant suffering - would being conscious have any value then? Kriegel (2019) also addresses these complex philosophical issues and proposes the following thought experiment, modelled after Siewert's: The choice you are now offered is not to be zombified (in exchange for a reward) or not, but rather to be zombified or to continue to live a life that "will skew constantly towards the unpleasant" (p. 10). Our intuition here is that most of us would choose the second option anyway: (Moderately) unpleasant phenomenal experience is better than none, which again suggests that experience has intrinsic value. Kriegel offers another interesting thought experiment based on Nozick's (1974) concept of an "experience machine". Imagine you can either enter the experience machine and continue to experience (hallucinate) life as you would were you not trapped in the machine, or to be zombified. As Kriegel puts it, it would be folly not to choose to get plugged in(to the Matrix).

While we do not aim here to further explore the depths of the philosophical debates that involve the notion of value, it seems to us that these different arguments and intuitions are at least suggestive that phenomenal experience indeed has intrinsic value: Barring exceptional circumstances, if given a choice, most of us would probably prefer to be a poor conscious agent than being a rich zombie, to experience life vs. failing to feel anything. In other words, we would be quite willing to give up many non-phenomenal goods just to keep phenomenal experience, even in conditions where those phenomenal experiences might not always be experiences we want to have.

We now turn to the notion of subjective value in experimental sciences. Subjective value is a central construct in at least three different domains: perceptual consciousness, decision making 
and emotion research. Indeed, paradigmatic phenomenal experiences, such as the taste of an apple or the warmth of an embrace, are described under different names and with emphasis on different aspects in all three domains. For thinkers interested in consciousness, such experiences constitute instances of mental states characterized by their distinctive qualia, the emphasis being placed on subjective aspects rather than on value, as discussed further below. For decision making researchers, they represent rewards to be obtained: while the notion of value is central to decision making, subjective aspects have been rather neglected. In affective research, they highlight the fact that emotional feelings colour almost every mental state or action, with an intensity reminiscent of value and that is also characterized by a subjective feel. Our point here is to suggest that these are all but mere renderings of the same core assumption, namely that subjective experience has value. In the following, we briefly overview how the notion of subjective value has been approached from these different experimental perspectives.

\section{Decision making}

In the field of decision making, subjective value classically refers to the quantity assigned to an item and that underlies choices and preference judgements: you choose whichever item has the highest value. Whether value is determined intrinsically by the participant (e.g., as when deciding whether to have red or white wine) or whether there is an objective consensus on what the best option may be (e.g., a reward of $10 €$ is more valuable than a reward of $1 €$ ) does not matter in most models of decision making: value is a number on a common scale, with different values characterized by rank order and a distance metric (Levy \& Glimcher, 2012).

Subjective value is considered subjective only inasmuch as it is subject-dependent, as in whether one prefers Bordeaux vs. Bourgogne. In other words, most of the relevant research has emphasized value in and of itself, leaving its subjective aspect remains mostly unaddressed (but see Azzalini, Buot, Palminteri, \& Tallon-Baudry, 2021). A recent trend in computational decision making relates value more directly to the internal state and intrinsic goals of the subject of experience: the value of a reward is computed as the distance between the current state and the state the agent would find herself in if she obtained the reward (Juechems \& Summerfield, 2019; Keramati \& Gutkin, 2014). However, despite massive progress in the development of computational models of decision making, the precise computation through which the reward function, that is, the function that associates an item with value, remains largely unspecified (Juechems \& Summerfield, 2019). In some cases, an item might have an obvious value, as defined by evolution, to satisfy basic needs (Keramati \& Gutkin, 2014). But how does one compute the subjective value that one spontaneously assigns to the taste of wine, since taste, as such, does not satisfy a basic need, and since the value associated with taste is distinct from the value associated with the calorific value of the wine? No rule of evolution specifies or even constrains this subjective taste value. Subjective values and resulting preferences are shaped by learning, of course, but the central question is why do we have subjective values at all? In the case of wine, we can easily imagine two wines with the same caloric content, one we like and the other we don't. What is the purpose of having different subjective values for two items that objectively fulfil the same basic need?

The conception of reinforcement learning algorithms illustrate the gap between rewards associated with a predefined value defined by the programmer (or evolution in the case of living organisms) and reward associated with intrinsic value. Thus, all reinforcement learning algorithms are driven by "rewards", but such rewards only have extrinsic value - they define what the system will do with respect to extrinsic constraints, but they have zero value for the system (i.e., AlphaGO zero) itself. Similarly, an intraveinous supply of water and nutrients has 
extrinsic value, and the caregiver knows that this nutrient supply is necessary to sustain his patient. However, a palatable meal has both an extrinsic, objective value, here related to caloric and nutrient content, and an intrinsic, subjective value related to its taste and smell that exists only because the meal is experienced. Subjective experience would thus contribute to the computation of the reward function by creating the link between an item and its subjective, intrinsic value. In other words, the key for a reward to have intrinsic value is precisely subjective experience, for in its absence, there is nobody for whom the reward means anything. How to design systems for which rewards have intrinsic value is a core challenge for future artificial intelligence research that we will not delve into beyond pointing out that for any system to want anything of its own, it has to have a sense of its own existence as an intentional agent and the ability to flexibly learn about its own internal states and its possibilities of actions.

\section{Emotions}

Emotions are an important drive for behavior: one seeks pleasurable experiences and attempts to avoid unpleasant ones. This can be viewed as a reformulation of decision making, where emotions have distinctive values that guide behavior - with little room for subjective experience. Indeed for some, emotions are only expressed through behavior, and can hence be objectively measured by the experimenter (Adolphs, Mlodinow, \& Barrett, 2019). Such emotional behaviors rely on survival circuits strongly constrained by evolution (Anderson \& Adolphs, 2014), generating a limited behavioral repertoire, such as freezing, aggression or avoidance. However, emotions in humans are also typically associated with rich and intense subjective feelings (Adolphs et al., 2019; LeDoux \& Hofmann, 2018). Emotional feelings can be viewed as a particular instance of subjective, phenomenal experience - what it feels like to savor an excellent wine, or to find pleasure, or maybe disgust, in an embrace, etc... But what is the function of emotional feelings? Clearly some behaviors convey an evolutionary advantage (don't be eaten, reproduce), but what is the function of joy, remorse or schadenfreude?

Whether and how emotional feelings are related to the neural activity in survival circuits is still unclear, but there is at least some degree of independence between the two. Indeed, those two components of emotions can predict different outcomes. Let us consider the example of basejumping: danger-avoidance should lead to never, ever base-jump. On the other hand, the exhilarating feeling of flying might have a high subjective value, speaking in favour of the risky behaviour of base-jumping. In other words, risky behaviour can be accounted for if the subjective experience of risky behaviour has intrinsic value that can override the value of safe behaviour selected by evolution.

While we are suggesting that an emotional subjective feeling is associated to a single value, so that it can be easily compared with something else, the characterization of subjective feelings in emotion research usually resorts to additional dimensions, from the two-dimensional space of valence and arousal (Russell \& Barrett, 1999) to more complex descriptions (Cowen \& Keltner, 2017). How a single value is constructed from a constellation of features is an open issue, even in the decision making literature (Pelletier \& Fellows, 2021; Pessiglione \& Daunizeau, 2021) and we refrain from venturing into any further.

\section{Conscious perception}

Consciousness research has focused on perception associated with phenomenal experience, the "what is it like" (Nagel, 1974) to taste an apple or to experience a red book. Qualia refer to 
specific characteristics of individual instances of conscious experience, or more prosaically " the way things seem to us" (Dennett, 1988). What is the point of seeing something consciously? We propose that perceptual experience is automatically associated with an intrinsic subjective value. Intuitively, we feel that seeing a painting or listening to birds has some worth - i.e., the very definition of value: the worth of something for someone. In the case of paintings, we are used to express our preferences - you might prefer a monochromatic painting by Yves Klein to one of the exquisitely detailed scenes of Jheronimus Bosch, or the reverse. The argument we make here is that this applies to all sensory conscious experiences: whenever you see, hear, touch, taste or smell something consciously, a subjectively value is automatically assigned, even if we do not think about such values as explicitly as in the case of paintings, and even if this value is close to zero.

Arguments in favor of an automatic assignment of value to a visual input can be found in the experimental literature on preferences. Preferences can be measured by asking participants to rate the likeability of images, hence providing a direct measure of the subjective value associated with the stimulus, or to choose between items, allowing the experimenter to infer preference values. Importantly, the brain automatically assigns a preference value to consciously experienced visual stimuli such as faces, houses, or paintings (Lebreton, Jorge, Michel, Thirion, \& Pessiglione, 2009), even when the task (judging age) was unrelated to the preference value measured in a separate session. Such findings directly support our hypothesis, which predicts that value assignment is an intrinsic feature of consciousness and that it occurs automatically. Automatic value assignment is precisely what endows conscious perceptual experience with a function. While the process of assigning a value to sensory inputs probably begins early in life and is fully automatized in adults, the specific value assigned to a given stimulus is likely to be modified by learning or habituation, and to be dependent on context the walk by the beach that you take every day might become less, or more, valuable with time, and be more, or less, pleasant depending on weather conditions.

The term value is mostly absent from the experimental consciousness literature. It might be that likeability is the final step in value computation, based on a collection of features, as proposed in the field of decision making (Pelletier \& Fellows, 2021; Pessiglione \& Daunizeau, 2021). Alternatively, value itself might be multi-dimensional. Despite all the work carried out on perceptual consciousness, the dimensions of conscious perceptual experience remain unknown. One's subjective experience of barely visible stimuli is often captured along a 4-point scale ranging from "Nothing" to "Clear experience" (Overgaard, Rote, Mouridsen, \& Ramsoy, 2006; Sandberg, Timmermans, Overgaard, \& Cleeremans, 2010), suggesting a link with intensity but also with clarity. At the other end of the spectrum, some studies have attempted to offer detailed analysis of subjective verbal reports (Petitmengin, van Beek, Bitbol, Nissou, \& Roepstorff, 2019), taking advantage of recent advances in automated language processing (Bedi et al., 2015), but such methods have not yet reached a point where they can reliably be used in most standard experimental paradigms. Nevertheless, a notion semantically related to value - the richness of perception - has been put forward in perceptual consciousness research. The richness of perception has initially been equated to the amount of details that can be retrieved from an image. This view has been particularly prominent in the debate on change blindness and inattentional blindness (Block, 2011b; Simons \& Levin, 1997), where participants miss prominent visual information such as a gorilla among basketball players. The inability to report substantial amounts of visual information stands in contrast with the subjective feeling that visual experience is rich, leading some authors to conclude that the richness of conscious experience is illusory (M. A. Cohen \& Dennett, 2011). However, this conclusion only holds if one equates richness with the quantity of details that can be retrieved. A different account for 
the experienced richness of perception might instead be found in the impression conveyed at a glance by a visual scene (Campana, Rebollo, Urai, Wyart, \& Tallon-Baudry, 2016; Campana \& Tallon-Baudry, 2013; Haun, Tononi, Koch, \& Tsuchiya, 2017) based on the rapid extraction of summary statistics (A. Cohen, Dennett, \& Kanwisher, 2016; Whitney \& Yamanashi Leib, 2018). In any case, the notion of value we put forward here is not related to the richness brought about by details: a monochromatic blue painting by Yves Klein induces - for some people - a valuable perceptual experience despite failing to contain any detail.

Associating value to phenomenal experience accounts for some puzzling experimental findings. For instance, navigating along a corridor is associated with both successful, functionally relevant behaviour, and a subjective experience that might be viewed as an epiphenomenon. Let's now turn to the case of TN, a blind patient with bilateral damage to primary visual cortex. Despite being blind, TN could nevertheless successfully navigate around obstacles in a long corridor (de Gelder et al., 2008). Importantly, despite being able to perform well, and despite being told of his ability, TN required guidance by another person to navigate in the environment in everyday life. In other words, the lack of subjective experience deprived TN of the willingness to engage in navigation. Conversely, more intense subjective perceptual experiences, such as described in alterations of visual perception induced by psilocybin, probably contribute to subjects reporting that "everything looks new, fascinating and more intense", even if those altered perceptions, often characterized by moving geometric shapes, have neither apparent meaning nor function (Bayne \& Carter, 2018; Preller \& Vollenweider, 2018)

The link between sensory experience and preferences is further connected to evolutionary considerations. Hoffman (2019) argued that perceptual systems need not capture reality "as it is" - they need only to improve a creature's fitness, that is, detect features of the world that are relevant to improving fitness. Thus, fitness trumps truth. At the evolutionary level, the point is simply to note that the sensory systems of different species are tailored to transduce those aspects of reality that optimally improve fitness. The evolution of sensory systems naturally leads to the emergence of inner representational spaces that are tailored to each species' ecological niche - von Uexküll's notion of "Umwelt" (Uexküll, 1957). This presumably also explains the origin of preferences, not only across species, but also across individuals. In this respect, Voltaire (1962 / 1764), in his Philosophical Dictionary, wrote: "Ask a toad what beauty is, the to kalon? He will answer you that it is his toad wife with two great round eyes issuing from her little head, a wide, flat mouth, a yellow belly, a brown back." Beauty is the eye of the beholder. What I take to be beautiful depends on preferences and biases that have accrued as a result of my experience with the world and with other people.

\section{Subjective experience, once endowed with value, is functionally relevant}

Having assigned value to subjective experience, we can now begin to provide answers to our key question: "What is the functional advantage, if any, of having subjective experience"?

\section{The value of subjective experience accounts for flexible, creative behaviour}

Assigning value to subjective experience accounts for flexible, creative behaviour. Conscious agents care about their experiences in ways that non-conscious systems do not. Simple organisms can act in adaptive ways installed by evolution; algorithms can act in appropriate ways programmed by design, and so on. However, the behavioural repertoire of conscious agents is much more extended. Humans create and design novel experiences, from playing 
music to sledging, from telling jokes to creating new cooking recipes. Subjective experience gives value to what has no intrinsic value as defined by the rules of physics or biology. An agent's ability to enjoy - or to loathe - conscious experience affords considerable adaptive value - the agent can now act in ways that flexibly dodge the constraints set up by evolution as it gives it much greater freedom to act in ways it chooses for its own reasons, and even in ways that are clearly counter-adaptive, as when one commits suicide to defend an idea, or engage in otherwise self-harmful behaviour. Conscious agents act upon themselves, upon the world, and upon other people because experiencing specific phenomenal states has intrinsic value.

\section{The value of subjective experience accounts for internal drive}

The value of subjective experience is a powerful internal drive for behaviour. Imagine you can make money but not use it. Would you spend any effort making money just to see the numbers go up on your accounts, especially if those numbers remain unknown to anyone but you ${ }^{1}$ ? Some would, but most would not, for, obviously, what would be the point? Value-driven algorithms, including reinforcement learning, work that way. Their only reason they act at all is because they are programmed to maximize a reward function, but paradoxically, the reward itself is not rewarding for them. This also holds for many biological organisms, who are programmed by evolution to survive, reproduce, and otherwise achieve homeostasis. In other words, obtaining rewards is a powerful drive for behaviour. But what defines a reward, beyond basic biological needs? If conscious experience has value, then generating conscious experience is rewarding. Because the reward function is internally generated rather than imposed by biological constrains, conscious agents act for reasons of their own. Seeking new conscious (hence valuable) experiences thus constitutes a drive to engage in behaviour that does not need to be justified by further reasons. Acting "just to see how it feels", "for fun" - such as a child pricking a needle in her palm or a tourist trying food that smells terribly bad - does not fulfil any basic need. While it may use the same neural routes and share the same algorithms than behaviour driven by basic needs, the motivation - the internal reward function - is very different. Unconscious agents such as algorithms can act, but they do not act for reasons of their own; for acting in one way or another has no import for them. Biological systems can be driven by goals that need not be conscious - survival, reproduction, and so on - but recognizing the value of subjective experience accounts for the drive of human beings to generate behaviour for reasons of their own. Conversely, zombies do not care about dying or about having an orgasm - since they lack conscious experience, nothing ever means anything to them and hence they completely lack motivation to do anything at all.

\section{Subjective values create a common currency for the mind}

Endowing each conscious experience not only with a specific phenomenal content but also with a value enables the comparison and ranking of conscious experiences. It is striking to see how humans are apt at analogy-making (French, 1995), which requires mapping concepts across vastly different semantic spaces (e.g., think of a pen as weapon). Likewise, most of us readily engage in seemingly complicated preference judgments such as "would you rather go for a walk with friends or stay home and read?" Those two experiences have little in common in terms of experiential features - one takes place outside, in a social context; the other indoors and in a solitary setting, but they can be easily compared in the common value space described in decision making (Lebreton et al., 2009; Levy \& Glimcher, 2012; Montague \& Berns, 2002)

\footnotetext{
${ }^{1}$ There is a striking computer game where the player does just that: https://www.decisionproblem.com/paperclips/index $2 . h t m l$
} 
Endowing conscious experience with value thus provides us with a unified space that probably contributes to the unity of consciousness. Value coding, if taking place in phenomenal space (i.e., "what does this do to $m e$ ?") is precisely the kind of mechanism one would expect to enable the emergence of a unified phenomenal space, essentially by creating a common, reward-based currency in the brain. Pushing this reasoning further, perhaps it is in virtue of the fact that all our experiences take place in a unified phenomenal space that functional features such as global accessibility emerge, rather than the opposite.

\section{The experiencing subject should be at the heart of theories of consciousness but also of decision making and emotions}

Value is defined as the worth of something for someone, echoing the definition of consciousness by Nagel: "an organism has conscious mental states if and only if there is something that it is like to be that organism - something it is like for that organism" (Nagel, 1974). Similarly, an emotional feeling exists only if there an organism to experience this feeling. In conscious perception research, the experimental measure of consciousness that has been mostly used is the report "I saw the stimulus" but only few studies attempted at identifying the mechanisms underlying the "I" part of the statement (Park, Correia, Ducorps, \& Tallon-Baudry, 2014). The experiencing subject should thus be at the heart of theories of (perceptual) consciousness but also of decision making and emotions. The experiencing subject requires one adopts a firstperson perspective (Azzalini, Rebollo, \& Tallon-Baudry, 2019), defined as a combination of a unified viewpoint (on both the external world and internal space) and intrinsic 'mineness' (Zahavi, 2005).

What is the biological substrate of the experiencing subject? The simplest definition of a self is related to the organism that needs to be fed, regulated and protected. Interestingly, there is a convergence of theories pertaining to the emotional self (Damasio, 2010), bodily self (Blanke \& Metzinger, 2009) or perceptual consciousness (Park \& Tallon-Baudry, 2014) to root the experiencing subject in the monitoring of bodily signals coming from joints and muscles (Blanke \& Metzinger, 2009) as well as from the viscera (Azzalini et al., 2019). However, many feeding, regulatory or protective behaviors can be accounted for without introducing the notion of consciousness, instead relying solely on circuits and values selected by evolution.

Under our hypothesis, consciousness would have evolved and been selected because it adds an important degree of freedom to the machinery of reward-based behavior: Behaviour that seems purposeless can nevertheless have intrinsic value, but this, crucially, only holds when associated with conscious experience. Subjective experience hence becomes the central drive for the discovery and creation of new behaviors. Under our hypothesis, observations such as crows sledging on a roof or dogs repeatedly dragging a plank up a snowy slope to enjoy the pleasure of speeding down on it thereafter, that is, behaviors that apparently serve no function beyond that of procuring enjoyment, might be taken as a sign of phenomenal consciousness. As we elaborate below, the transition between behaviour driven mostly by circuits designed through evolution and novel, creative behaviour is likely to be a gradual, rather than an abrupt one. While our hypothesis would be in favour of animal consciousness, at least to some degree, it also suggests a close association between consciousness and life, with consequences for the possibility of artificial consciousness or of consciousness in lab-grown brains (Reardon, 2020). As we have briefly discussed above, the problem with contemporary AI systems is not with further augmenting their computational prowess, but rather to design them in a way that they want things for reasons of their own. 


\section{Conclusion: Dissolution of the hard problem?}

The distinction between access-consciousness and phenomenal consciousness has been at the core of many debates in the consciousness literature. Some have argued that there are distinct neural correlates of each (Block, 2005), others have offered arguments against that view (B. Baars \& Laureys, 2005). There has also been substantial debate about the richness of phenomenal experience, in particular about whether experience overflows access, that is, about the extent to which we experience more than we can tell. Overflow theorists (e.g., Block, 2011a) have argued that it does while others have been highly critical (e.g., Phillips, 2011, 2015). We will refrain from further discussion of such debates because doing so would be derivative to the two core points we wanted to emphasize with this article:

The first is that the distinction between phenomenal and access consciousness, as productive as it has been driving robust debate in consciousness research, is fundamentally misleading. On the one hand it has led some to defend the idea that phenomenal experience is but a mere epiphenomenon that actually plays no functional role in our existence - a perspective that flies in the face of our own experience as human beings. On the other hand, it has led some authors to defend the idea that there can be phenomenal experience in the absence of access, that is, that phenomenal experience is entirely independent from the machinery necessary for access (Fahrenfort \& Lamme, 2012). It is against this view that Cohen \& Dennett (2011) have argued that "the hard problem is an impossible problem", by which they mean to assert that "dissociative" theories of consciousness, that is, theories that distinguish between access and phenomenology, are fundamentally unfalsifiable and hence unscientific. Cohen and Dennett proposed the following "perfect experiment" — disconnect the entire set of brain areas responsible for color perception (V4 in particular) and ask participants what they experience when shown an apple and asked to report on its color. Cohen and Dennett speculate that participants would report not perceiving color at all, despite the fact that the isolated V4 continues to function normally (M. A. Cohen \& Dennett, 2012), while Fahrenfort and Lamme insist that since V4 would continue to function normally, "the parsimonious account is to infer that perceptual states continue to exist without access".

Here, what strikes us is how this discussion turns out to completely eschew the subject whose experiences or lack thereof form the core of the debate. What could it possibly mean for an isolated cortical region to experience anything at all, even though its neural activity would be congruent with "normal" perception? Phenomenal experiences, and mental states in general, presuppose, as Frege argued, the existence of a subject who can experience those states. It is here, perhaps, that the social world acquires the importance it has for humans. There are clear functional advantages for an organism to be capable of ascertaining the unobservable mental states of other agents, be they conspecifics or predators. Inferring other agents' mental states is a predictive problem that requires model building. While successfully predicting the consequences of action on the world is relatively easy, successfully predicting the consequences of actions directed towards other agents is difficult: It requires us to make assumptions about the unobservable mental states that drive other agents' response to my own actions - their state of mind, their intentions, their mood, their personality, and so on. In other words, this is where theory of mind comes to play a central role. The core argument here is that the machinery necessary to successfully infer the mental states of other agents leverages the same mechanisms as those necessary to engage in the sort of reflexive redescription that we think is involved in self-awareness. That is, if phenomenal experience indeed depends on the involvement of systems of metarepresentations that redescribe and qualify first-order representations, as Higher-Order Thought Theory suggests (Brown et al., 2019; Fleming, 2019), then such 
metarepresentational systems will be shaped by, and possibly emerge from our predictiondriven interactions with other agents. As argued in (Cleeremans et al., 2020), "something unique happens when a developing agent has models of itself available to it (Carruthers, 2009) in the form of other agents that it can infer the unobservable internal states of merely by interacting with them (Tamir \& Thornton, 2018; Thornton, Weaverdick, \& Tamir, 2019). Selves are thus embodied, virtual and transparent renditions of the underlying biological machinery (Metzinger, 2003) that produces them, and emerge progressively over development as a mandatory consequence of dynamic interactions with other agents (Frith, 2007; Shea et al., 2014)."

If phenomenal experience presupposes the existence of a subject, as we have argued, it might also be necessary for access, for there would not be anything for a subject to access unless it were experienced. Congruently, Clark (2000) argued that there are cases where access implies qualia. Clark offers a contrast between two possible responses a system can give when asked to report on the differences between two visual stimuli (say, a difference in colour). The system can either, Clark argues, report that "the answer just comes to it" (as in blindsight), or that it is "non-inferentially aware that it is using the visual vs., say, the tactile modality to make its judgment, which is what normal agents do. But in that latter case, says Clark, the system "must say that there is something it is like to see the difference rather than e.g. to smell it.". In other words, Clark continues, at least in such cases, "certain facts about patterns of access (the "easy" stuff) can actually imply facts about phenomenality and "what it's like". Clark concludes that this excludes the possibility of zombies, for "honest reports of genuine direct, non-inferential access to acts of perceptual difference detection imply the presence of genuine phenomenal differences" (p. 33).

And thus, our second core point, which admittedly goes much beyond the debates about the relationships beween access consciousness and phenomenal consciousness: We surmise that the very function of consciousness is precisely to endow organisms with phenomenal experience. In other words, what consciousness does is to make it possible for organisms to experience themselves, the world, and other agents. This perspective places the experiencing subject at the heart of consciousness research and can perhaps result in a dissolution of the hard problem: Phenomenal states, rather than being functionless - a mysterious ingredient added to a cognitive soup - instead constitute the very basis upon which we do everything that we do. And conversely, we probably wouldn't do much at all were it not for the fact that we experience things. To quote Dennett (personal communication) again: "How do we go from doing things for reasons to having reasons for doing things?". Having reasons for doing things is precisely what consciousness enables.

ACKNOWLEDGMENTS: AC is a Research Director with the F.R.S.-FNRS (Belgium). Both AC and CTB are fellows of the Canadian Institute for Advanced Research (CIFAR) program "Brain, Mind and Consciousness". CTB is supported by the European Research Council (ERC) under the European Union's Horizon 2020 research and innovation program (grant agreement No 670325, Advanced grant BRAVIUS) and ANR-17-EURE-0017. 


\section{REFERENCES}

Adolphs, R., Mlodinow, L., \& Barrett, L. F. (2019). What is an emotion? Curr Biol, 29(20), R1060R1064. doi:10.1016/j.cub.2019.09.008

Anderson, D. J., \& Adolphs, R. (2014). A framework for studying emotions across species. Cell, 157(1), 187-200. doi:10.1016/j.cell.2014.03.003

Azzalini, D., Buot, A., Palminteri, S., \& Tallon-Baudry, C. (2021). Responses to heartbeats in ventromedial prefrontal cortex contribute to subjective preference-based decisions. Journal of Neuroscience.

Azzalini, D., Rebollo, I., \& Tallon-Baudry, C. (2019). Visceral Signals Shape Brain Dynamics and Cognition. Trends Cogn Sci, 23(6), 488-509. doi:10.1016/j.tics.2019.03.007

Baars, B., \& Laureys, S. (2005). One, not two neural correlates of consciousness. Trends in Cognitive Sciences, 9(6), 269.

Baars, B. J. (1988). A Cognitive Theory of Consciousness. Cambridge: Cambridge University Press.

Bayne, T. (2013). Agency as a marker of consciousness. In A. Clark, J. Kiverstein, \& T. Vierkant (Eds.), Decomposing the will (pp. 160-177). Oxford: Oxford University Press.

Bayne, T., \& Carter, O. (2018). Dimensions of consciousness and the psychedelic state. Neuroscience of consciousness, 2018(1).

Bedi, G., Carrillo, F., Cecchi, G. A., Slezak, D. F., Sigman, M., Mota, N. B., . . Corcoran, C. M. (2015). Automated analysis of free speech predicts psychosis onset in high-risk youths. NPJ Schizophr, 1, 15030. doi:10.1038/npjschz.2015.30

Berthoud, H. R. (2011). Metabolic and hedonic drives in the neural control of appetite: who is the boss? Curr Opin Neurobiol, 21(6), 888-896. doi:10.1016/j.conb.2011.09.004

Blanke, O., \& Metzinger, T. (2009). Full-body illusions and minimal phenomenal selfhood. Trends Cogn Sci, 13(1), 7-13. doi:10.1016/j.tics.2008.10.003

Block, N. (1995). On a confusion about a function of consciousness. Behavioral and Brain Sciences, $18,227-287$.

Block, N. (2005). Two neural correlates of consciousness. Trends in Cognitive Science, 9(2), 46-52.

Block, N. (2011a). Perceptual consciousness overflows cognitive access. Trends in Cognitive Sciences, $15,567-575$.

Block, N. (2011b). Perceptual consciousness overflows cognitive access. Trends Cogn Sci, 15(12), $567-$ 575. doi:10.1016/j.tics.2011.11.001

Brown, R., Lau, H., \& LeDoux, J. E. (2019). Understanding the higher-order approach to consciousness. Trends in Cognitive Science, 23(9), 754-768.

Campana, F., Rebollo, I., Urai, A., Wyart, V., \& Tallon-Baudry, C. (2016). Conscious Vision Proceeds from Global to Local Content in Goal-Directed Tasks and Spontaneous Vision. J Neurosci, 36(19), 5200-5213. doi:10.1523/JNEUROSCI.3619-15.2016

Campana, F., \& Tallon-Baudry, C. (2013). Anchoring visual subjective experience in a neural model: the coarse vividness hypothesis. Neuropsychologia, 51(6), 1050-1060. doi:10.1016/j.neuropsychologia.2013.02.021

Carruthers, P. (2009). How we know our own minds: the relationship between mindreading and metacognition. Behavioral and Brain Sciences, 32(2), 121-138.

Chalmers, D. J. (1995). Facing up to the problem of consciousness. Journal of Consciousness Studies, 2(3), 200-219.

Clark, A. (2000). A case where access implies qualia? Analysis, 60(1), 30-37.

Cleeremans, A. (2011). The radical plasticity thesis: How the brain learns to be conscious. Frontiers in Psychology, 2, 1-12.

Cleeremans, A. (2014). Connecting conscious and unconscious cognition. Cognitive Science, 38(6), 1286-1315.

Cleeremans, A., Achoui, D., Beauny, A., Keuninckx, L., Martin, J.-R., Muñoz-Moldes, S., . . . De Heering, A. (2020). Learning to be conscious. Trends in Cognitive Science, 24(2), 112-123.

Cohen, A., Dennett, D. C., \& Kanwisher, N. (2016). What is the bandwidth of perceptual experience? Trends in Cognitive Science, 20(5), 324-335. 
Cohen, M. A., \& Dennett, D. C. (2011). Consciousness cannot be separated from function. Trends Cogn Sci, 15(8), 358-364. doi:S1364-6613(11)00125-2 [pii]

10.1016/j.tics.2011.06.008

Cohen, M. A., \& Dennett, D. C. (2012). Response to Fahrenfort and Lamme: definiinf reportability, accessibility, and sufficiency in conscious awareness. Trends in Cognitive Science, 16(3), 139140.

Cowen, A. S., \& Keltner, D. (2017). Self-report captures 27 distinct categories of emotion bridged by continuous gradients. Proc Natl Acad Sci $U$ S A, 114(38), E7900-E7909. doi:10.1073/pnas.1702247114

Damasio, A. (2010). Self comes to mind: constructing the conscious brain. New York: Pantheon Books. de Gelder, B., Tamietto, M., van Boxtel, G., Goebel, R., Sahraie, A., van den Stock, J., . . Pegna, A. (2008). Intact navigation skills after bilateral loss of striate cortex. Curr Biol, 18(24), R11281129. doi:10.1016/j.cub.2008.11.002

Dehaene, S., Kerszberg, M., \& Changeux, J. P. (1998). A neuronal model of a global workspace in effortful cognitive tasks. Proceedings of the National Academy of Sciences of the U.S.A., 95(24), $14529-14534$.

Dennett, D. C. (1988). Quining Qualia. In A. J. Marcel \& E. Bisiach (Eds.), Consciousness in modern Science. Oxford, U.K.: Oxford University Press.

Dennett, D. C. (2016). Illusionism as the obvious default theory of consciousness. Journal of Consciousness Studies, 23(11-12), 65-72.

Dennett, D. C. (2019). Welcome to strong illusionism. Journal of Consciousness Studies, 26(9-10), 4858.

Doyen, S., Klein, O., Pichon, C.-L., \& Cleeremans, A. (2012). Bejaviorual priming: It's all in the mind, but whose mind? . PLOS One, 7(1), e29081.

Fahrenfort, J. J., \& Lamme, V. A. F. (2012). A true science of consciousness explains phenomenology: comment on Cohen and Dennett. Trends in Cognitive Science, 16(3), 138-139.

Fleming, S. M. (2019). Awareness reports as inference in a higher-order state space. arXiv:1906.00728 [q-bio]. Retrieved from http://arxiv.org/abs/1906.00728

Frankish, K. (2016). Illusionism as a theory of consciousness. Journal of Consciousness Studies, 23(1112), 11-39.

Frege, G. (1918). The thought: A logical enquiry. Mind, 65(259), 289-311.

French, R. M. (1995). The subtelty of sameness: A theory and comptuer model of analogy-making. Boston, MA.: MIT Press.

Frith, C. D. (2007). Making up the mind. Oxford: Blackwell Publishing.

Hassin, R. R., Uleman, J. S., \& Bargh, J. A. (2005). The new unconscious. Oxford, U.K.: Oxford University Press.

Haun, A. M., Tononi, G., Koch, C., \& Tsuchiya, N. (2017). Are we underestimating the richness of visual experience? Neurosci Conscious, 2017(1), niw023. doi:10.1093/nc/niw023

Hoffman, D. D. (2019). The case against reality: How evolution hid the truth from our eyes. New York: W.W. Norton

Juechems, K., \& Summerfield, C. (2019). Where Does Value Come From? Trends Cogn Sci, 23(10), 836-850. doi:10.1016/j.tics.2019.07.012

Keramati, M., \& Gutkin, B. (2014). Homeostatic reinforcement learning for integrating reward collection and physiological stability. Elife, 3. doi:10.7554/eLife.04811

Kriegel, U. (2004). The functional role of consciousness: A phenomenological approach. Phenomenology and the Cognitive Sciences, 3, 171-194.

Kriegel, U. (2019). The value of consciousness. Analysis, 79, 503-520.

Lau, H., \& Rosenthal, D. (2011). Empirical support for higher-order theories of consciousness. Trends in Cognitive Sciences, 15(8), 365-373.

Lebreton, M., Jorge, S., Michel, V., Thirion, B., \& Pessiglione, M. (2009). An automatic valuation system in the human brain: evidence from functional neuroimaging. Neuron, 64(3), 431-439.

LeDoux, J. E., \& Hofmann, S. G. (2018). The subjective experience of emotion: a fearful view. Current Opinion in Behavioral Sciences, 19, 67-72. doi:10.1016/j.cobeha.2017.09.011

Lee, A. Y. (2019). Is consciousness intrinsically valuable? Philosophical Studies, 175, 1-17. 
Levy, D. J., \& Glimcher, P. W. (2012). The root of all value: a neural common currency for choice. Curr Opin Neurobiol, 22(6), 1027-1038. doi:10.1016/j.conb.2012.06.001

Metzinger, T. (2003). Being No One: The self-model theory of subjectivity. Cambridge, MA: Bradford Books, MIT Press.

Montague, L. M., \& Berns, G. S. (2002). Neural economic and the biological substrates of valuation. Neuron, 36, 265-284.

Nagel, T. (1974). What is it like to be a bat? The Philosophical Review, 83, 435-450.

Newell, B. R., \& Shanks, D. (2014). Unconscious influences on decision making: A critical review. Behavioral and Brain Sciences, 37(1), 1-19.

Nozick, R. (1974). Anarchy, state, and utopia. New York: Basic Books.

O’Donnell, M., Nelson, L. D., Ackermann, E., Aczel, B., Akhtar, A., Aldrovandi, A., . . Zrubka, M. (2018). Registered replication report: Dijksterhuis and van Knippenberg (1998). Perspectives on Psychological Science, 13(2), 268-294.

Overgaard, M., Rote, J., Mouridsen, K., \& Ramsoy, T. Z. (2006). Is conscious perception gradual or dichotomous? A comparison of report methodologies during a visual task. Conscious Cogn, 15(4), 700-708. doi:S1053-8100(06)00031-6 [pii]

10.1016/j.concog.2006.04.002

Pacherie, E. (2008). The phenomenology of action: A conceptual framework. Cognition, 107, 179-217.

Park, H. D., Correia, S., Ducorps, A., \& Tallon-Baudry, C. (2014). Spontanerous fluctuations in neural responses to heartbeats predict visual detection. Nature Neuroscience, 17, 612-618.

Park, H. D., \& Tallon-Baudry, C. (2014). The neural subjective frame: from bodily signals to perceptual consciousness. Philos Trans $R$ Soc Lond B Biol Sci, 369(1641), 20130208. doi:10.1098/rstb.2013.0208

Pelletier, G., \& Fellows, L. K. (2021). Viewing orbitofrontal cortex contributions to decision-making through the lens of object recognition. Behavioral Neuroscience.

Pessiglione, M., \& Daunizeau, J. (2021). Bridging across functional models: the OFC as a value-making neural network. . Behavioral Neuroscience.

Peters, M. A., Kentridge, R., Phillips, I., \& Block, N. (2017). Does unconscious perception really exist? Continuing the ASSC20 debate. . Neuroscience of consciousness, 3(1), 1-11.

Peters, M. A., \& Lau, H. (2015). Human observers have optimal introspective access to perceptual processes even for visual masked stimuli. Elife, 4. doi:10.7554/eLife.09651

Petitmengin, C., van Beek, M., Bitbol, M., Nissou, J. M., \& Roepstorff, A. (2019). Studying the experience of meditation through Micro-phenomenology. Curr Opin Psychol, 28, 54-59. doi:10.1016/j.copsyc.2018.10.009

Phillips, I. (2011). Perception and iconic memory. Mind and Language, 26(381-411).

Phillips, I. (2015). No watershed for overflow: Recent work on the richness of consciousness. Philosophical Psychology, 236-249.

Phillips, I. (2021). Scepticism about unconscious perception is the defauly hypothesis. Journal of Consciousness Studies, 28(3-4).

Preller, K. H., \& Vollenweider, F. X. (2018). Phenomenology, structure and dynamics of psychedelic state. Current Topics in Behavioral Neurosciences, 36, 221-256.

Reardon, S. (2020). Can lab-grown brains become conscious? Nature, 586(7831), 658-661. doi:10.1038/d41586-020-02986-y

Robinson, Z., Maley, C. J., \& Piccinini, G. (2015). Is consciousness a spandrel? Journal of the American Philosophical Association, 1(2), 365-383.

Rosenthal, D. (1997). A theory of consciousness. In N. Block, O. Flanagan, \& G. Güzeldere (Eds.), The Nature of Consciousness: Philosophical Debates. Cambridge, MA: MIT Press.

Russell, J. A., \& Barrett, L. F. (1999). Core affect, prototypical emotional episodes, and other things called emotion: dissecting the elephant. J Pers Soc Psychol, 76(5), 805-819. doi:10.1037//00223514.76.5.805

Sandberg, K., Timmermans, B., Overgaard, M., \& Cleeremans, A. (2010). Measuring consciousness: is one measure better than the other? Consciousness and Cognition, 19(4), 1069-1078.

Seager, W. E. (2001). Consciousness, value and functionalism. Psyche 7((20)).

Searle, J. R. (1992). The Rediscovery of the Mind. Cambridge, MA.: MIT Press.

Seth, A. (2018). Functions of consciousness. PsyArXiv Preprints. 
Shanks, D. R., Green, R. E. A., \& Kolodny, J. A. (1994). A critical examination of the evidence for unconscious (implicit) learning. In C. Umiltà \& M. Moscovitch (Eds.), Attention and performance $X V$ : Conscious and nonconscious information processing (pp. 837-860). Cambridge: MIT Press.

Shea, N., Boldt, A., Bang, D., Yeung, N., Heyes, C., \& Frith, C. D. (2014). Supra-personal cognitive control and metacognition. Trends in cognitive sciences, 18(186-193).

Siewert, C. (1998). The significance of consciousness. Princetion, N.J. (USA): Princeton University Press.

Silver, D., Hubert, T., Schrittwieser, J., Antonoglou, I., Lai, M., Guez, A., . . Hassabis, D. (2018). A general reinforcement learning algorithm that masters chess, shogi and go through self-play. Science, 362(6419), 1140-1144.

Silver, D., Schrittwieser, J., Simonyan, K., Antonoglou, I., Huang, A., Guez, A., . . Hassabis, D. (2017). Mastering the game of Go without human knowledge. Nature, 550, 354. doi:10.1038/nature24270

Simons, D. J., \& Levin, D. T. (1997). Change blindness. Trends Cogn Sci, 1(7), 261-267. doi:10.1016/S1364-6613(97)01080-2

Tamir, D., \& Thornton, M. A. (2018). Modeling the predictive social mind. Trends in Cognitive Science, 22(3), 201-212.

Thornton, M. A., Weaverdick, M. E., \& Tamir, D. I. (2019). The social brain automatically predicts other's future mental states. Journal of Neuroscience, 39(1), 140-148.

Tye, M. (1996). The function of consciousness. Noûs, 30(3), 287-305.

Uexküll, J. v. (1957). A stroll through the worlds of animals and men: A picture book of invisible worlds. In C. H. Schiller (Ed.), Instinctive behavior: The development of a modern concept. New York: International Universities Press.

Voltaire. (1962 / 1764). A philosophical dictionary: Basic Books.

Weiskrantz, L. (1986). Blindsight: A case study and implications. Oxford, England: Oxford University Press.

Whitney, D., \& Yamanashi Leib, A. (2018). Ensemble Perception. Annu Rev Psychol, 69, 105-129. doi:10.1146/annurev-psych-010416-044232

Zahavi, D. (2005). Subjectivity and selfhood: investigating the first-person perspective. Cambridge, Massachussets: MIT press.

Zimmerman, M. J. (2019). Intrinsic vs. extrinsic value. In N. Zalta (Ed.), The Stanford Encyclopedia of Philosophy. 